Psychother Psychosom 2019;88:52-54

DOI: $10.1159 / 000495045$

\title{
Introducing Network Intervention Analysis to Investigate Sequential, Symptom-Specific Treatment Effects: A Demonstration in Co-Occurring Insomnia and Depression
}

\author{
Tessa F. Blanken ${ }^{\text {a Tanja Van Der Zweerde }}{ }^{\text {b }}$ \\ Annemieke Van Straten ${ }^{\mathrm{b}}$ Eus J.W. Van Someren ${ }^{\mathrm{a}, \mathrm{c}, \mathrm{d}}$ \\ Denny Borsboom ${ }^{\text {e Jaap Lancee }}{ }^{f}$

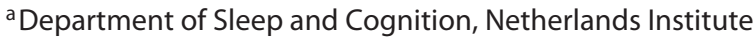 \\ for Neuroscience, an Institute of the Royal Netherlands \\ Academy of Arts and Sciences, Amsterdam, The Netherlands; \\ ${ }^{b}$ Department of Clinical Psychology, Amsterdam Public Health \\ Research Institute, Vrije Universiteit Amsterdam, Amsterdam, \\ The Netherlands; ' Department of Integrative Neurophysiology, \\ Center for Neurogenomics and Cognitive Research, Vrije \\ Universiteit Amsterdam, Amsterdam, The Netherlands; \\ ${ }^{\mathrm{d}}$ Amsterdam UMC, Vrije Universiteit Amsterdam, Psychiatry, \\ Amsterdam, The Netherlands; ${ }^{\text {}}$ Department of Psychological \\ Methods, University of Amsterdam, Amsterdam, \\ The Netherlands; ${ }^{f}$ Department of Clinical Psychology, \\ University of Amsterdam, Amsterdam, The Netherlands
}

\section{Dear Editor,}

Insomnia and depression are among the most prevalent mental disorders and often co-occur [1]. Insomnia is a primary risk factor for the development of depression [2]. In people with depression, insomnia impedes remission [3, 4], while cognitive-behavioral therapy for insomnia (CBTI) accelerates it $[5,6]$. However, overlap in the symptoms of insomnia and depression obscures the sequential development and order of treatment-induced changes: do CBTI-induced sleep improvements precede alleviation of depression symptoms, or does CBTI affect depression directly?

A possibility to investigate the effect of CBTI on insomnia and depression while taking symptom overlap into account is offered by network models that conceptualize mental disorders as networks of symptoms structured by their associations [7]. Here we introduce Network Intervention Analysis (NIA) as an extension of these models to follow the development of treatment-induced changes in symptoms and their association structure over time, while distinguishing direct and indirect effects.

We applied NIA to identify the sequential development and order of CBTI-induced effects on symptoms of insomnia and depression throughout the course of treatment. We used data from a randomized controlled trial [6] in which participants with symp-

T.F. Blanken and T. Van Der Zweerde contributed equally to this work.

\section{KARGER}

E-Mail karger@karger.com www.karger.com/pps
(C) 2019 The Author(s)

Published by S. Karger AG, Basel

Karger

Open access

This article is licensed under the Creative Commons AttributionNonCommercial-NoDerivatives 4.0 International License (CC BYNC-ND) (http://www.karger.com/Services/OpenAccessLicense). Usage and distribution for commercial purposes as well as any distribution of modified material requires written permission.
Fig. 1. The estimated regularized networks correspond to the 10 assessment weeks ( 2 before treatment (T0, T1), 5 during treatment (T2-T6), 3 after treatment (T7-T9); left to right, top to bottom). The networks include the Insomnia Severity Index and Patient Health Questionnaire items (circles) and treatment (square). The edges represent the conditional dependence relations among the variables that capture the unique associations among the variables, while controlling for all the other variables in the network. Green edges represent positive associations, red edges represent negative associations, and the thickness and color saturation of the edge is proportional to the strength of the association. The size of the node is proportional to the difference in symptom severity between the treatment and control group, where smaller node sizes represent greater differences in favor of the treatment group. For each node, the proportion of explained variance by the other nodes in the network, i.e., the predictability, is visualized by a ring around each node: a completely filled ring indicates that $100 \%$ of the variance is explained, a completely empty ring corresponds to an explained variance of $0 \%$. Treatment is directly related to "early morning awakenings" (T2, T4, T7, T9), "difficulty maintaining sleep" (T3, T4, T6-T9), "suicidal thoughts" (T3, T5), "dissatisfaction with sleep" (T5-T7, T9), "psychomotor agitation" (T7)*, or "worry about sleep" (T9). * Note that this edge is partly obscured by the edge between treatment and "dissatisfaction with sleep." However, a small edge was retrieved in the network.

(For figure see next page.) toms of insomnia and depression received either 5 weeks of CBTI $(n=52)$ or no treatment $(n=52)$. Symptoms of insomnia (Insomnia Severity Index) and depression (Patient Health Questionnaire) ment T0-T1, during treatment T2-T6, after treatment T7-T9). included a treatment allocation variable in addition to all symptoms. This procedure allowed us not only to follow the sequential
development of treatment-induced changes in the severity of individual symptoms, but also to distinguish, at each point in time, the specific symptoms that were most directly affected by treatment sequential development of treatment-induced changes in the network structure of associations by estimating, for each symptom, in the network (called predictability). Details on sample characteristics and the NIA are provided in the online supplementary matesuppl. material)

ic treatment effects throughout 10 weeks (animated version: Figure.gif). The edges visualize the unique associations between variables, controlling for their associations with all the other vari-

$$
\text { (2) }
$$




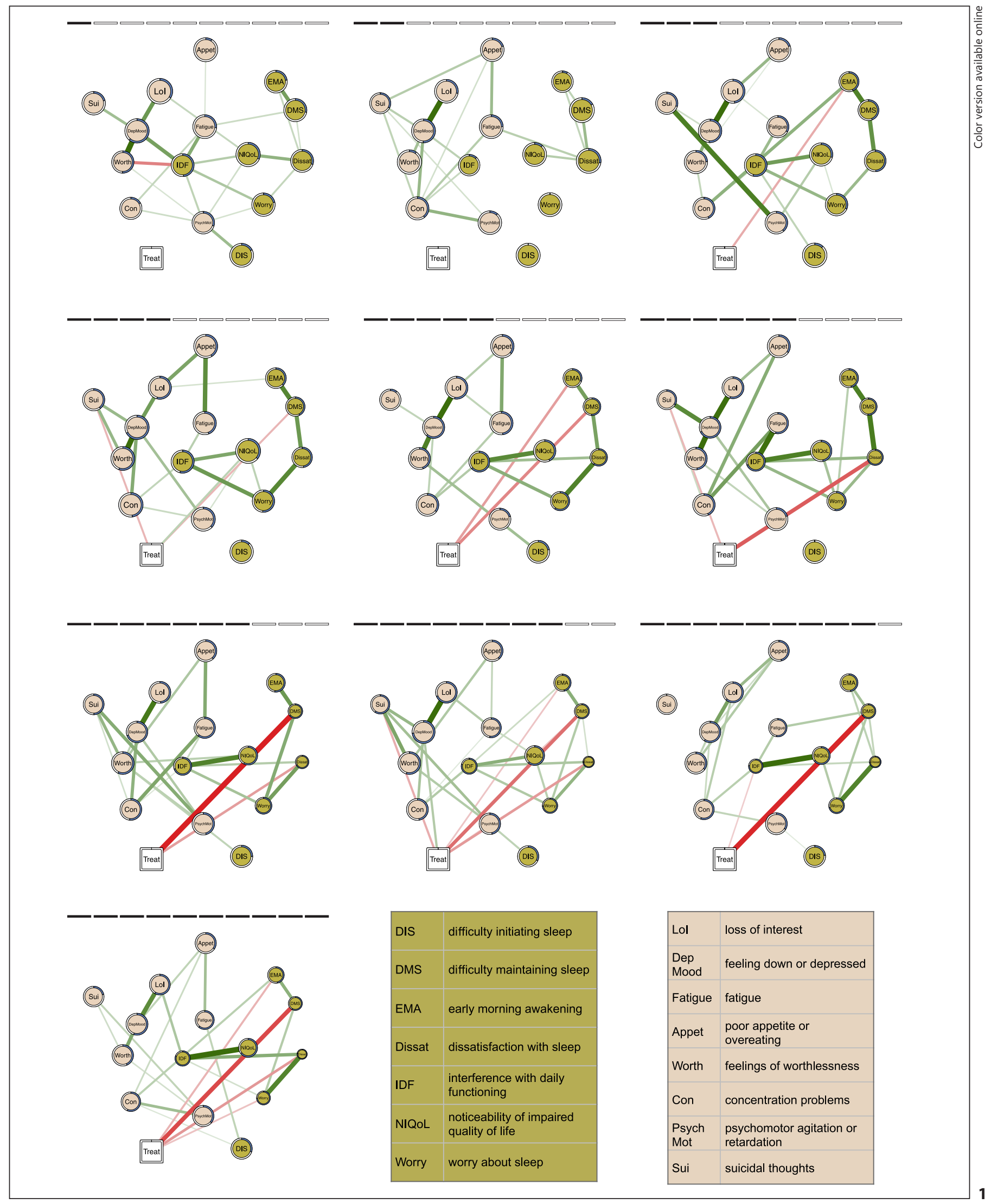

Network Intervention Analysis to Investigate Treatment Effects 
ables (i.e., conditional dependence relationships). Since the variable "treatment" can influence the symptom variables, but not vice versa, an edge between the treatment node and a symptom identifies the symptoms that are directly affected by treatment. The effect of treatment on each symptom relative to changes observed in controls is visualized in the size of the nodes.

First, the edges between the treatment and symptom variables revealed a direct effect of treatment primarily on insomnia symptoms. Effects commenced already after the first week of treatment and continued after completion of the intervention. The sequential development indicated that treatment first and most consistently improved the sleep problems "early morning awakening" (assessments T2, T4, T7, and T9) and "difficulty maintaining sleep" (first moderately during $\mathrm{T} 2$ and $\mathrm{T} 3$, and then increasingly so from T6 to T9). Only after 4 weeks of treatment did "dissatisfaction with sleep" improve (T5-T7 and T9), indicating a sequential effect of treatment on sleep problems first and dissatisfaction later. NIA moreover indicated that the direct effect of treatment on these specific symptoms secondarily "spread" through the network via their associations with other symptoms. For example, one of the strongest treatment effects was on "worry about sleep," even though it was directly affected by treatment on only one of the assessments (T9).

Second, NIA allowed us to observe how treatment ignited changes in the associations among the symptoms. For each symptom we inspected whether the amount of variance explained by the other symptoms (i.e., predictability) changed during treatment. The predictability systematically and robustly increased over time from, on average, $30 \%$ at baseline to, on average, 53\% after assessment. There was no systematic increase or decrease in symptom variance over the course of treatment that drove the increase in predictability $(r=0.10)$. The finding suggests that treatment alters the structure of the network, which is in line with the network theory of mental disorders [7] and earlier findings [8].

We demonstrated NIA as a method to investigate the sequential process of symptom-specific direct and indirect effects of treatment. The use was illustrated by elucidating direct and indirect effects of CBTI on symptoms of insomnia and depression. Across all assessments, CBTI most strongly and directly affected specific sleep complaints. Accordingly, in the current sample of people suffering from insomnia and subclinical depression, the likely route for CBTI to improve symptoms of depression is indirect, through its effects on two dominant sleep complaints.

One previous study incorporated a treatment-allocation variable in network analysis [9], but only to determine the relative efficacy of two treatments on individual symptoms of depression in a pre-post comparison. A particular strength of the current NIA approach is that it additionally elucidates the sequential order of direct and indirect treatment-induced symptom improvements. Some limitations merit consideration. As in any multivariate approach, "directness" of effects should be interpreted cautiously if unmeasured variables are likely to be involved. Specific to our study, small treatment effects and weak associations may have gone undetected due to the moderate sample size and the use of LASSO regularization to ensure high specificity [10]. The absence of a direct edge should not be interpreted as an absence of any effect, but rather as an indication that an indirect effect is more likely given the available data. NIA can be considered as a statistical "searchlight" to identify plausible trajectories of treatment-induced effects.

NIA offers a technique to investigate the sequential development and order of treatment-induced changes of specific symp- toms, highlighting likely pathways through which treatment effects evolve. Use of this technique can aid to understand treatment mechanisms and reveal clues to their optimization.

\section{Acknowledgments}

The authors would like to thank Dr. Marieke Effting for her valuable input in this study and Jonas Haslbeck for his help using his mgm R-package.

\section{Disclosure Statement}

The authors have no conflicts of interest to declare.

\section{Funding Sources}

This work was supported financially by the European Research Council (grant number ERC-ADG-2014-671084-INSOMNIA) and by a VU University Research Fellowship 2016-2017.

\section{Author Contributions}

T. Van Der Zweerde and J. Lancee carried out recruitment and data collection and coordinated the randomized controlled trial from which the data stem. T.F. Blanken, T. Van Der Zweerde, and J. Lancee designed the current study. T.F. Blanken performed the network analyses and interpretation of the results, supervised by D. Borsboom. T.F. Blanken and T. Van Der Zweerde drafted the manuscript. All authors contributed to and approved the manuscript.

\section{References}

1 Taylor DJ, Lichstein KL, Durrence HH, Reidel BW, Bush AJ. Epidemiology of insomnia, depression, and anxiety. Sleep. 2005 Nov;28(11):1457-64.

2 Baglioni C, Battagliese G, Feige B, Spiegelhalder K, Nissen C, Voderholzer $\mathrm{U}$, et al. Insomnia as a predictor of depression: a meta-analytic evaluation of longitudinal epidemiological studies. J Affect Disord. 2011 Dec; 135(1-3):10-9.

3 Thase ME, Buysse DJ, Frank E, Cherry CR, Cornes CL, Mallinger AG, et al. Which depressed patients will respond to interpersonal psychotherapy? The role of abnormal EEG sleep profiles. Am J Psychiatry. 1997 Apr; 154(4):502-9.

4 Chan JW, Lam SP, Li SX, Yu MW, Chan NY, Zhang J, et al. Eveningness and insomnia: independent risk factors of nonremission in major depressive disorder. Sleep (Basel). 2014 May;37(5):911-7.

5 Ballesio A, Aquino MR, Feige B, Johann AF, Kyle SD, Spiegelhalder K, et al. The effectiveness of behavioural and cognitive behavioural therapies for insomnia on depressive and fatigue symptoms: A systematic review and network meta-analysis. Sleep Med Rev. 2018 Feb;37:114-29.

6 Van der Zweerde T, Van Straten A, Effting M, Kyle S, Lancee J. Does online insomnia treatment reduce depressive symptoms? A randomized controlled trial in individuals with both insomnia and depressive symptoms. Psychol Med. 2018 May 11:1-9. doi: 10.1017/S0033291718001149 [Epub ahead of print].

7 Borsboom D. A network theory of mental disorders. World Psychiatry. 2017 Feb;16(1):5-13.

8 Fried EI, van Borkulo CD, Epskamp S, Schoevers RA, Tuerlinckx F, Borsboom D. Measuring depression over time ... Or not? Lack of unidimensionality and longitudinal measurement invariance in four common rating scales of depression. Psychol Assess. 2016 Nov;28(11):1354-67.

9 Bekhuis E, Schoevers R, de Boer M, Peen J, Dekker J, Van H, et al. Symptom-specific effects of psychotherapy versus combined therapy in the treatment of mild to moderate depression: A network approach. Psychother Psychosom. 2018;87(2):121-3.

10 Epskamp S, Fried EI. A tutorial on regularized partial correlation networks. Psychol Methods. 2018 Mar 29. doi: 10.1037/met0000167 [Epub ahead of print]. 